\title{
Breast enlargement in Malawian males on the standard first-line antiretroviral therapy regimen: Case reports and review of the literature
}

\section{A Kwekwesa ${ }^{1}$, C Kandionamaso ${ }^{1}$, N Winata ${ }^{1,2}$, E Mwinjiwa ${ }^{1}$, M Joshua ${ }^{3}$, D Garone $^{1}$, R Bedell', $^{\prime}$ JJ van Oosterhout ${ }^{1,4}$}

1. Dignitas International, Zomba, Malawi

2. University of Calgary, Calgary, Canada

3. Zomba Central Hospital, Ministry of Health, Zomba, Malawi

4. Department of Medicine, College of Medicine, Blantyre, Malawi

Correspondence to: Joep J. van Oosterhout

E-mail: joepvanoosterhout@gmail.com

\section{Case presentations}

1. A 35-year-old man was referred to Zomba Central Hospital in June 2014 from a health center with a presenting complaint of enlarged breasts. This had started gradually, approximately six months prior. He did not experience any pain or discharge from his nipples. He had no other complaints and felt generally healthy. In early 2013 he had been concomitantly diagnosed with pulmonary tuberculosis and HIV infection and was started on cotrimoxazole prophylaxis and antiretroviral therapy (ART) with tenofovir, lamivudine, and efavirenz, which is the standard first-line adult ART regimen, known in Malawi as regimen 5A. At that point, he had WHO clinical stage III HIV disease; a CD4 count was not done. There was no further relevant past medical history. He looked healthy and had normal vital signs. The rest of the physical examination was normal except for bilateral, symmetrical, elastic, and concentric swelling of the breasts, without discrete nodules, skin changes, or lymphadenopathy in the axillae and neck. There were no features of lipodystrophy and no stigmata of chronic liver disease. The genital examination was normal, without palpable abnormalities of the normal-sized testes. Upon laboratory investigation, his CD4 count was 130 cells/ $\mu \mathrm{L}$ and a full blood count was normal apart from the presence of macrocytosis. Liver enzymes were unremarkable.

2. In October 2014, we saw a 56-year-old male who presented with development of painless bilateral breast enlargement. This had started in May 2014, followed by spontaneous regression and then subsequent worsening from August 2014. The left breast was more conspicuously affected. He also mentioned experiencing weight loss but had no other symptoms. He was diagnosed with HIV infection in 2005 and started on ART in 2008 due to a low CD4 count of 83 cells $/ \mu \mathrm{L}$. While on his first ART regimen-stavudine, lamivudine, and nevirapine-he experienced progressive peripheral neuropathy and was eventually switched to a regimen of zidovudine, lamivudine, and nevirapine, after which his CD4 count was 409 cells/ $\mu \mathrm{L}$ and plasma HIV-1 RNA was undetectable. Six months later, in January 2010, he developed severe anaemia, secondary to zidovudine, and his ART regimen was changed again, this time to tenofovir, lamivudine, and efavirenz (regimen 5A). He had no other significant past medical history and the only medications he used were cotrimoxazole prophylaxis and tenofovir, lamivudine, and efavirenz. Bilateral breast enlargement (left more than right, see Figure 1) was the only abnormality found on physical examination, with the same pertinent negative findings as in the first case presented in this report. Figure 1: Gynaecomastia in patient 2

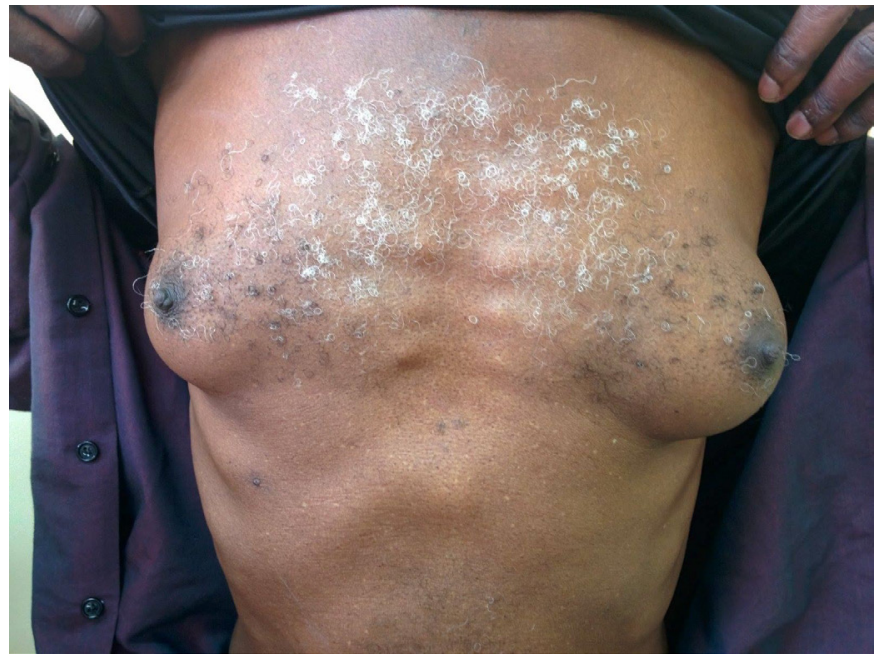

\section{Discussion}

Both patients had gynaecomastia, which is defined as a benign proliferation of breast tissue in males. It has to be distinguished from pseudogynecomastia and lipomastia, which are both forms of fat accumulation in the breasts. Lipomastia is part of the lipodystrophy syndrome. Antiretroviral drugs that commonly cause lipomastia include stavudine, didanosine, and protease inhibitors. Breast palpation can help to distinguish fat accumulation in the breasts from true gynaecomastia. ${ }^{1}$ In gynaecomastia, typically a soft, elastic or firm disk-shaped mass, that is concentric with the nipple-areola, can be palpated with the patient lying flat on his back. Uncommonly, benign tumours and breast cancer can also cause breast enlargement in males.

Once the presence of gynaecomastia is established, a differential diagnosis of its causes needs to be considered. There are two broad categories: physiological gynaecomastia (common) and pathological gynaecomastia (uncommon). Physiological gynaecomastia is self-limiting and occurs in newborns, adolescents, and the elderly. The pathological causes can be further divided up into three subcategories, namely testosterone deficiency, increased oestrogen production, and drug-induced breast enlargement. Testosterone deficiency can occur in HIV infection. Causes of excess oestrogen include $\beta$-hCG-producing tumours (especially testicular cancers), chronic liver disease, and malnutrition. Drugs that can induce gynaecomastia and are commonly used in Malawi include ketoconazole, cimetidine, omeprazole, spironolactone, isoniazid, calcium channel blockers, ACE inhibitors, and antiretroviral drugs. If the cause of gynaecomastia remains unknown, it is classified as idiopathic. A comprehensive overview of the pathological causes of gynaecomastia is shown in Table $1 .{ }^{1}$

We conducted a PubMed search with the search terms HIV and gynaecomastia and located 71 publications, none being from Africa. The oldest case report was in an 
HIV-infected patient in 1987, before the introduction of antiretroviral drugs. ${ }^{4} \mathrm{~A}$ French study noted the prevalence of gynaecomastia in patients on ART for more than two years to be $2.8 \% .{ }^{5}$ In Switzerland, the incidence of gynaecomastia in patients on ART was $2.3 \%$. The first case series documenting efavirenz-associated gynaecomastia was published in $2001 .^{6}$ A case-control study from New York $(n=23)$ described an association between gynaecomastia and efavirenz. ${ }^{7}$ In Spain, a case-control study $(\mathrm{n}=30)$ found an association with efavirenz, but also with didanosine and hypogonadism, with resolution occurring in $75 \%$ of the cases. ${ }^{8}$ In a case series $(n=5)$ of efavirenz-associated gynaecomastia, the onset of gynaecomastia occurred between 4 and 15 months after the start of ART. Upon a change of regimen, resolution was seen in all cases after several months. ${ }^{9}$ In vitro experiments have suggested that efavirenz activates oestrogen receptors in breast tissue, ${ }^{10}$ although subsequent experiments to confirm this finding have not yet been conducted. A systematic review concluded that the quality of the evidence of the association between gynaecomastia and antiretroviral drugs was moderate in general, being most convincing with efavirenz. ${ }^{11}$ We found one paper, from Nigeria, that was not included in PubMed, with six well-documented cases of efavirenz-associated gynaecomastia, of which five resolved upon stopping efavirenz. ${ }^{12}$

The literature demonstrates that gynaecomastia is a rare but well-documented side effect of efavirenz, but data from Malawi are lacking and only a single report exists from the whole of Africa. The incidence, prevalence, and risk factors of efavirenz-associated gynecomastia in Africans are thus unknown. Routine side effect monitoring of ART in Malawi's HIV programme strongly underreports the true prevalence of toxicities. Although there are sections for side effects in electronic monitoring systems that are used in larger HIV clinics and on the paper master cards used in smaller HIV clinics, clinicians often do not report side effects unless they are planning a change in ART regimen..$^{13}$ In addition, gynaecomastia is not explicitly listed as a side effect in routine monitoring and evaluation tools. Clinicians will therefore not be reminded that efavirenz causes gynaecomastia and may forget to screen patients for it during busy HIV clinics. Even if clinicians do remember to screen for gynaecomastia, they will be unable to record it as a specific side effect. As a result, gynaecomastia does not appear in Malawi's national ART statistics. Moreover, patients may not be educated on all the ART side effects and may not be aware that the gynaecomastia they experience is caused by their ART regimen.

Genetic factors that differ between races and ethnic groups impact on drug toxicities. ${ }^{14}$ Extrapolation of findings from Western settings may therefore lead to overestimation or underestimation of side effects in Africans. Genetic polymorphisms, particularly in genes coding for cytochrome P450 2B6 and other liver enzymes involved in the metabolism of efavirenz, are well-documented in African populations. ${ }^{15}$ Some are known to lead to reduced efavirenz metabolism, higher drug levels, and more neurotoxicity, and could also be relevant for gynaecomastia occurring among men taking efavirenz.

After the introduction of the new standardized first-line ART regimen 5A, which contains efavirenz, there were several case reports in the South East Health Zone of Malawi documenting gynaecomastia in patients who had changed from the previous to the new first-line regimen.
Articles were published in Malawian newspapers and on websites describing it as common and debilitating, especially in the Mangochi district. This created some controversy and concerns arose that the media attention could affect male enrolment in HIV care. Although gynaecomastia is a fairly rare side effect that is not physically harmful for the individual, it may thus have a wider negative public health impact, as it could lead to reduced linkage to ART and increased non-adherence and defaulting from ART among men.

We propose practical measures to manage the issue of gynaecomastia in Malawi. At a public health level, we advise that gynaecomastia be included in standard pre-ART counselling. Males should receive the following information: gynaecomastia is a rare side effect of ART that is not harmful but should be reported at an early stage, when a change of ART regimen will have a better opportunity to promote spontaneous resolution. Secondly, gynaecomastia should be added to the electronic monitoring systems, master cards, and standard lists that nurses use for screening for and reporting of side effects. On an individual level, if a patient has developed breast swelling, it should first be established whether it is true gynaecomastia, mainly by physical examination. Then causes of gynaecomastia other than efavirenz should be considered, such as testicular tumours, malnutrition, chronic liver disease, and other drugs. If efavirenz is found to be the most likely cause of the gynaecomastia, reassurance and explanation should be offered with special attention to adherence, which may be at risk. A change of ART regimen to tenofovir, lamivudine, and nevirapine (Malawi ART regimen 6A) should generally be made and resolution should be monitored over the course of several months. Surgery is only indicated in severe cases with severe cosmetic distress and when resolution does not occur in the months following change of ART regimen.

Both of our patients changed from efavirenz to nevirapine. Six months later patient 1 reported some improvement and patient 2 said the situation was stable. They were satisfied with their condition despite the limited subjective and objective reduction of the gynaecomastia. The incomplete resolution may be related to the advanced condition that had been present for several months before efavirenz was stopped. Alternatively, the gynaecomastia may have resulted from HIV-associated low testosterone levels.

\section{Conclusion}

Gynaecomastia is a well-recognized side effect of efavirenz that has cosmetic and psychological consequences. Its incidence in Africans is currently unknown. Awareness by patients and clinicians and early management by substitution of efavirenz with nevirapine can lead to resolution over months. Appropriate counselling should be offered to patients with gynaecomastia to avoid ART interruptions and non-adherence. 
Table 1: Causes of gynaecomastia ${ }^{\ddagger}$

\begin{tabular}{|c|c|c|c|c|}
\hline 1. Physiological & 2. Pathological & & & \\
\hline Newborns & 2a. testosterone deficiency & 2b. increased oestrogen production & 2c. drugs & \\
\hline \multirow[t]{31}{*}{ Adolescents } & \multirow{4}{*}{$\begin{array}{l}\text { Rare congenital syndromes ( } 5 \alpha \text {-reductase } \\
\text { deficiency, Klinefelter syndrome, androgen } \\
\text { insensitivity syndrome, congenital } \\
\text { anorchia) }\end{array}$} & Testicular tumours & Alcohol & Methadone \\
\hline & & & Alkylating agents & Methotrexate \\
\hline & & Lung carcinoma & Amiodarone & Methyldopa \\
\hline & & \multirow{3}{*}{$\begin{array}{l}\text { Other } \beta \text {-hCG-producing tumours, such as } \\
\text { renal cell cancer }\end{array}$} & Amlodipine & Metoclopramide \\
\hline & \multirow{2}{*}{ Viral orchitis, HIV } & & Amphetamines & Metronidazole \\
\hline & & & Anabolic steroids & Minocycline \\
\hline & Trauma, torsion, castration & Adrenal disease and tumours & $\begin{array}{l}\text { Angiotensin-converting } \\
\text { enzyme inhibitors }\end{array}$ & $\begin{array}{l}\text { Minoxidil } \\
\text { Mirtazapine }\end{array}$ \\
\hline & Renal failure & Chronic liver disease & Antiretroviral agents & Nifedipine \\
\hline & \multirow{2}{*}{ Haemochromatosis } & \multirow{2}{*}{ Malnutrition } & Atorvastatin & Nilutamide \\
\hline & & & Bicalutamide & Oestrogens and oestrogen- \\
\hline & \multirow{21}{*}{ Neurological, granulomatous disease } & \multirow[t]{21}{*}{ Hyperthyroidism } & Cimetidine & stimulating drugs \\
\hline & & & Cisplatin & Omeprazole \\
\hline & & & Diazepam & Paroxetine \\
\hline & & & Digoxin & Penicillamine \\
\hline & & & Didanosine & Phenothiazines \\
\hline & & & Diltiazem & Phenytoin \\
\hline & & & Efavirenz & Phytoestrogens \\
\hline & & & Etomidate & Protease inhibitors \\
\hline & & & Fenofibrate & Ranitidine \\
\hline & & & Finasteride & Reserpine \\
\hline & & & Fluoxetine & Risperidone \\
\hline & & & Flutamide & Rosuvastatin \\
\hline & & & Gonadotropin-releasing & Spironolactone \\
\hline & & & hormone agonists & Sulindac \\
\hline & & & Haloperidol & Stavudine \\
\hline & & & Heroin & Theophylline \\
\hline & & & Human chorionic & Tricyclic antidepressants \\
\hline & & & gonadotropins & Venlafaxine \\
\hline & & & Isoniazid & Verapamil \\
\hline & & & Ketoconazole & Vinca alkaloids \\
\hline & & & Marijuana & \\
\hline
\end{tabular}

${ }^{\mp}$ Adapted from Braunstein ${ }^{1}$, Johnson et al. ${ }^{2}$, and Dickson ${ }^{3}$

\section{References}

1. Braunstein GD. Gynecomastia. N Engl J Med. 2007; 357(12): 1229 1237.

2. Johnson RE, Murad MH. Gynecomastia: Pathophysiology, Evaluation, and Management. Mayo Clin Proc. 2009; 84(11): 10101015.

3. Dickson, G. Gynecomastia. Am Fam Physician. 2012; 85(7): 716722.

4. Couderc LJ, Clauvel JP. HIV-Infection-Induced Gynecomastia. Ann Intern Med. 1987; 107(2): 257.

5. Piroth L, Grappin M, Petit JM, et al. Incidence of gynecomastia in men infected with HIV and treated with highly active antiretroviral therapy. Scand J Infect Dis. 2001; 33: 559-560.

6. Caso JA, Prieto JM, Casas E, Sanz J. Gynecomastia without lipodystrophy syndrome in HIV-infected men treated with efavirenz. AIDS. 2001; 15: 1447-1448.

7. Rahim, S, Ortiz O, Maslow M, et al. A case-control study of gynecomastia in HIV-1 infected patients receiving HAART. AIDS Read. 2004; 14: 23-4, 29-32, 35-40.

8. Mira JA, et al. Gynaecomastia in HIV-infected men on highly active antiretroviral therapy: association with efavirenz and didanosine treatment. Antiviral Therapy. 2004; 9: 501-517.

9. Jover F, Cuadrado JM, Roig P, Rodriguez M, Andreu L, Merino J. Efavirenz-associated gynecomastia: report of five cases and review of the literature. Breast J. 2004; 10:244-246.
10. Sikora MJ, Rae JM, Johnson MD, Desta Z. Efavirenz directly modulates estrogen receptor and induces breast cancer cell growth. HIV Med. 2010; 11 (9): 603-607.

11. Deepinder F, Braunstein GD. Drug-induced gynecomastia: an evidence-based review. Expert Opin Drug Saf. 2012; 11(5): 779-95.

12. Abaji OO, Agaba PA, Ekeh PN, et al. Efavirenz-induced gynecomastia in HIV-infected Nigerian men: A report of six cases. Journal of Medicine and Medical Sciences. 2011; 2(11): 1221-1224.

13. Tapsfield J, Mathews T, Lungu M, van Oosterhout JJ. Underreporting of side effects of standard first-line ART in the routine setting in Blantyre, Malawi. Malawi Med J. 2011; 23(4):115-7.

14. Ngaimisi E, Habtewold A, Minzi O, Makonnen E, et al. Importancce of Ethnicity, CYP2B6 and ABCB1 Genotype for Efavirenz Pharmacokinetics and Treatment Outcomes: A Parallel-Group Prospective Cohort Study in Two Sub-Saharan Africa Populations. PLoS ONE. 2013; 8(7):e67946. doi: 10.1371/journal.pone.0067946.

15. Burger D, Van Der Heiden I, La Porte C, et al. Interpatient variability in the pharmacokinetics of the HIV non-nucleoside reverse transcriptase inhibitor efavirenz: the effect of gender, race and CYP2B6 polymorphism. British Journal of Clinical Pharmacology. 2005; doi: 10.1111/j.1365-2125.2005.02536.x. 